Interfaces

\title{
« Derrière un grillage » d'Octave Mirbeau et l'Hommage des artistes à Picquart
}

\section{Nadia Fartas}

\section{(2) OpenEdition}

1 Journals

Édition électronique

URL : http://journals.openedition.org/interfaces/672

DOI : 10.4000/interfaces. 672

ISSN : 2647-6754

Éditeur :

Université de Bourgogne, Université de Paris, College of the Holy Cross

\section{Édition imprimée}

Date de publication : 12 décembre 2019

Pagination : 165-188

ISSN : 1164-6225

\section{Référence électronique}

Nadia Fartas, " «Derrière un grillage » d'Octave Mirbeau et l'Hommage des artistes à Picquart », Interfaces [En ligne], 42 | 2019, mis en ligne le 12 décembre 2019, consulté le 06 janvier 2021. URL: http://journals.openedition.org/interfaces/672; DOI : https://doi.org/10.4000/interfaces.672

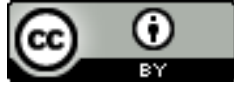

Les contenus de la revue Interfaces sont mis à disposition selon les termes de la Licence Creative Commons Attribution 4.0 International. 


\title{
« DERRIÈRE UN GRILLAGE » D'OCTAVE MIRBEAU ET L'HOMMAGE DES ARTISTES À PICQUART
}

\author{
Nadia Fartas \\ Docteure de l'EHESS-Université Paris-Sorbonne
}

Cet article est issu d'une communication présentée à la session «Ekphrasis : Unfolding the Visual » organisée par Heidrun Führer et Miriam Vieira dans le cadre du Congrès 2017 de l'Association Internationale pour l'Étude des Rapports entre Texte et Image (AIERTI-IAWIS) intitulé « La reproduction des images et des textes » (université de Lausanne, 10-14 juillet 2017).

\section{Résumé}

En janvier 1899, la Société libre d'édition des gens de lettres publie un album grand format de douze lithographies en l'honneur du lieutenant-colonel Georges Picquart alors emprisonné en raison de ses révélations en faveur de l'innocence du capitaine Alfred Dreyfus. Hommage des artistes à Picquart contient deux autres contributions : une préface d'Octave Mirbeau intitulée « Derrière un grillage » et une importante liste de protestataires qui affirment eux aussi leur soutien au colonel. On se propose de considérer la manière dont le recours à l'évidence vient appuyer le principe de justice qui traverse l'ensemble de cette publication entre le verbal et le visuel. Dans son texte l'écrivain cherche en effet à rendre manifeste la situation d'isolement et d'injustice qui frappe Picquart. Les artistes s'attachent également dans leur œuvre à soutenir l'innocence du colonel. Suivre le fil de l'évidence permet ainsi de souligner le primat de la lumière dans les lithographies de l'album qui font usage de l'allégorie de la Vérité, tandis que le principe de vérité est menacé dans le moment décisif de la III ${ }^{e}$ République qu'est la décennie 1890. Or l'idée même de justice, son exigence, sont au fondement de l'idée républicaine, si ce n'est de la politique. Dès lors, apparaît la forme originale d'engagement par l'art et la littérature que constitue l'Hommage des artistes à Picquart au moment où en France s'affirme la figure de l'intellectuel associé au dreyfusard. C'est que l'album ainsi constitué retourne contre l'État le principe qui le fonde et qu'il est censé défendre : la justice. 


\begin{abstract}
In January 1899, the Société libre d'édition des gens de lettres published a large format album of twelve litographs in honour of lieutenant-colonel Georges Picquart: then in jail because of his revelations in favour of the innocence of Captain Alfred Dreyfus. There are two more contributions in Hommage des artistes à Picquart: a preface by Octave Mirbeau entitled "Derrière un grillage" and a significant list of protestors who, they too, claimed their support to the colonel. We propose to consider the way resorting to evidence supports the principle of justice which goes through the whole publication between the verbal and the visual. Indeed, in this text, the writer tries to make the isolation and injustice situation Picquart was being struck obvious. The artists were also concerned with sustaining the innocence of the colonel in their works. Following the thread of evidence thus enables to underline light primacy in the litographs of the album which make use of the allegory of Truth while the principle of Truth was being threatened at the decisive moment of the IIIrd Republic which is the 1890 decade. Now, the idea of justice, its requirement, are at the foundations of the republican idea, if not of politics. That being the case, what is shown is the original form of commitment through art and literature which the Hommage des artistes à Picquart constitutes at the time when the figure of the intellectual associated to the defender of Dreyfus was asserting itself in France. It is that the album thus built up turns back against the state the very principle that founds it and that it is supposed to defend : justice.
\end{abstract}

En janvier 1899, la Société libre d'édition des gens de lettres publie, en trois cents exemplaires, un album grand format de douze lithographies ${ }^{87}$ en l'honneur du lieutenant-colonel Georges Picquart alors emprisonné en raison de ses révélations en faveur de l'innocence du capitaine Alfred Dreyfus ${ }^{88}$. Hommage des artistes à Picquart contient deux autres contributions : d'une part, une préface de

87 Les trois cents exemplaires sont répartis comme suit : « 20 exemplaires de collaborateurs, sur Japon impérial, épreuves signées (numérotés de 1 à 20) ; 30 exemplaires de grand luxe, sur Japon impérial, épreuves signées, à 100 francs l'exemplaire (numérotés de 21 à 50) ; 250 exemplaires de souscripteurs, lithographies sur Hollande à la forme, texte sur pur alfa, à 20 francs l'exemplaire (numérotés de 51 à 300) ». Dimensions : 40,5 x $31 \mathrm{~cm}$. Les illustrations de cet article sont issues de l'exemplaire n²00 conservé à la Bibliothèque de l'Institut National d'Histoire de l’Art à Paris (Fléjou et Poulain). Les lithographies sont reproduites dans Kleeblatt 216-221.

88 Le lieutenant-colonel Georges Picquart a rapproché lécriture du commandant Ferdinand Walsin-Esterhazy de celle présente sur « le bordereau » qui valut au capitaine Dreyfus d’être injustement accusé de trahison. Picquart sera incarcéré le 13 juillet 1898 et libéré le 13 juin 1899, c’est-à-dire deux mois avant le second procès de Dreyfus à Rennes. 
l'écrivain et critique Octave Mirbeau ${ }^{89}$ intitulée « Derrière un grillage », et d'autre part, une importante liste de protestataires ${ }^{90}$ — entre trente et quarante mille signatures (Charle 142) - qui affirment eux aussi leur soutien au colonel. Dans son texte Mirbeau cherche à rendre évidente la situation d'isolement et d'injustice qui frappe Picquart. Les douze artistes, parmi lesquels se trouvent les plus fervents peintres et dessinateurs du camp dreyfusards (Luce, Vallotton), s'attachent également dans leur œuvre à défendre l'innocence du colonel. ${ }^{91}$ Le statut singulier de cette préface peut-il dès lors être interrogé par le biais de l'ekphrasis ? En effet, s'il convient davantage d'utiliser le terme de description ${ }^{92}$ pour aborder les parties du discours qui dans un texte de l'époque contemporaine réfèrent à une œuvre d'art ou bien à des notations visuelles, puisque l'ekphrasis désigne un exercice codifié de la rhétorique antique, l'une des propriétés de l'ekphrasis s'avère toutefois particulièrement pertinente pour analyser l'objet singulier que constitue l'album. Il s'agit de l'enargeia, autrement dit, comme le souligne Bernard Vouilloux, de la " vivacité visuelle (vividness en anglais) », de " l'évidence visuelle » qu'implique l'ekphrasis, celle-ci désignant, selon la définition qu'en donne Hermogène, " un discours détaillé qui rend, pour ainsi dire, évident et qui met sous les yeux ce que l'on veut montrer » (22). À côté de l'aspect sémiotique engagé par la description l'enargeia répond ainsi à l'aspect cognitif de l'ekphrasis (Le Tournant « artiste $» 37)$.

Or il n'est question que de l'évidence dans cet album, puisqu'il s'agit pour les différents acteurs et énonciateurs de défendre avec force l'innocence du colonel Picquart, ceci dans le cadre plus large de la demande de révision du procès du capitaine Dreyfus. C'est pourquoi je m'interrogerai moins sur le statut de la préface de Mirbeau - ce texte constitue-t-il ou non une ekphrasis - que sur la manière dont l'évidence vient appuyer le principe de justice qui traverse l'ensemble de cette publication, entre le verbal et le visuel. Suivre le fil de l'enargeia permet ainsi d'insister sur le primat de la lumière, corollaire de la clarté inhérente à l'enargeia, dans les lithographies de l'album qui font usage de l'allégorie de la Vérité, alors que le principe de vérité est menacé dans le moment décisif de la III République qu'est la décennie 1890. Or l'idée même de justice, son exigence, sont au fondement de

89 La préface est reproduite dans Mirbeau (239-244), édition à laquelle nous nous référons. Paul Brunet et Félix Thureau sont à lorigine de cette publication et de cette invitation faite à Mirbeau décrire un texte, celui-ci ayant visité plusieurs fois le colonel Picquart en prison depuis son arrestation le 13 juillet 1898. Voir Mirbeau 33 et 242.

90 Du 25 novembre au vendredi 9 décembre 1898 (pages 24-168 dans l'édition).

91 Nous n'avons pas trouvé trace d’une commande spécifique faite aux auteurs des lithographies.

92 Voir à ce sujet les travaux de Bernard Vouilloux et notamment Le Tournant « artiste » (chapitre premier, « Descriptions ») 
l'idée républicaine, si ce n'est de la politique. La justice sociale est en outre revendiquée par le socialisme et particulièrement par Jaurès (Duclert, Jaurès... 21-23) qui a lui aussi défendu Picquart dans ses écrits. Dès lors, apparaît la forme originale d'engagement par l'art et la littérature que constitue l'Hommage des artistes à Picquart au moment où en France s'affirme la figure de l'intellectuel associé au dreyfusard. C'est que l'album retourne contre l'État le principe qui le fonde et qu'il est censé défendre : la justice.

\section{La Préface-plaidoyer de Mirbeau : dénoncer l’inversion des principes républicains}

On sait que dans sa critique d'art, où priment « le dialogue pamphlétaire et la fiction narrative anecdote, conte - comme vecteurs d'émotions "), au regard notamment de la peinture impressionniste, Mirbeau s'avère peu adepte de l'ekphrasis (Bat 109). On peut en revanche constater que cette technique sert la visée de l'auteur : " Derrière un grillage » entend aborder la question de la justice. "Écorché vif, écrivent Pierre Michel et Jean-François Nivet, Mirbeau souffre littéralement de toutes les formes d'injustice » (344). C'est pourquoi, dans son oeuvre, le critique entend « démystifier » la loi afin de démontrer que « l'injustice, l'arbitraire et l'exploitation sont tapis au cœur même du système juridique : faite par les riches et pour les riches, la loi justifie tous les abus et toutes les extorsions » (260) analysent les deux historiens à propos de la pensée de Mirbeau. Dans cette préface particulière, puisqu'elle n'introduit pas un texte ${ }^{93}$ mais des images et une liste de protestataires, Mirbeau en vient ainsi à dénoncer une véritable inversion des principes républicains. C'est pourquoi "Derrière un grillage » s'offre comme un puissant plaidoyer.

Trois parties distinctes composent la préface. Le ton dominant renvoie tout d'abord à la sphère judiciaire. Le plaidoyer s'ouvre sur une dénonciation virulente :

Voilà plus de six longs mois que le colonel Picquart est en prison. Il est en prison pour avoir refusé de s'associer à un crime ; il est en prison pour avoir crié l'innocence d'un homme, condamné au pire des supplices ; il est en prison pour avoir voulu cette chose, aujourd'hui proscrite de toute la vie : la justice. [...] Voilà plus de six longs mois que le colonel Picquart est en prison et les traîtres, eux, sont bien tranquilles. [...] Par une étonnante perversion du sens patriotique, ils sont protégés, défendus, acclamés. Ils reçoivent l'accolade des princes.

93 « Je nommerai ici préface, par généralisation du terme le plus fréquemment employé en français, toute espèce de texte liminaire (préliminaire ou postliminaire), auctorial ou allographe, consistant en un discours produit à propos du texte qui suit ou qui précède » (Genette 164). 
Le paradoxe est affirmé par le fait que la justice n'est plus considérée comme un principe universel dans la $\mathrm{III}^{\mathrm{e}}$ République. Il est pourtant bien contraire à l'idée de la république de pratiquer et de soutenir la corruption. Ce monde inversé, où les traîtres sont à la lumière, concerne aussi les intellectuels : « Des écrivains, des artistes, des philosophes, de hauts fonctionnaires, des bâtonniers fraternisent avec eux... » (239-240) Mirbeau s'en distingue avec force et le portrait élogieux qu'il fait de Picquart, " épris de justice », en empruntant à la figure évangélique du Christ outragé, laquelle comptait parmi les figures zoliennes, ${ }^{94}$ assoit son propre courage de citoyen et d'homme de lettres. De la sorte Mirbeau se présente comme un humaniste convaincu. Le portrait élogieux du colonel vient ainsi donner du relief à la dénonciation :

Le colonel Picquart avait le choix, entre la plus belle carrière qui se fût jamais ouverte devant un officier, et le cachot. On ne lui demandait que de se taire. Il a préféré parler et, de ce fait, il a choisi le cachot. [...] Avec ce calme admirable, ce courage ferme et tranquille qu'il met dans tous les actes de sa vie, il est venu se livrer à ses ennemis (241).

Puis dans un deuxième temps l'auteur se présente face à Picquart. La notion d'évidence devient l'objet même de la description qui s'offre au lecteur par le biais du regard (« Je l'ai vu »). L'idée de tableau est manifeste par le titre de la préface : "Derrière un grillage » suggère un plan et un cadre, lesquels s'ajoutent aux barreaux qu'implique le cachot. L'écrivain souligne l'aspect obscur de cette pièce, d'où va pourtant émerger la vérité incarnée par Picquart. Le processus qui mène à la rencontre des deux hommes s'accomplit dans un cheminement qui conduit au-delà des obstacles constitués par les barreaux et la grille :

Le parloir est sombre. Le jour entre à peine, dans cette pièce où par l'unique fenêtre qui l'éclaire, le regard se heurte au mur très haut, très noir, infiniment triste de la prison. Le prisonnier est au fond du parloir, entre des barreaux, dans une sorte de cage, comme une bête, séparé du visiteur par un assez large couloir grillagé. L'impression est vraiment pénible. Les mailles du double grillage sont si fines, que, tout d'abord, on ne voit rien. Puis, peu à peu, on perçoit une figure indécise, qui va et vient sur le grillage, une figure lointaine, presque sans contours, une figure effacée, comme une forme qui s'enfonce dans la brume... (242)

Le confus domine dans cet emboîtement d'espaces (prison, parloir, couloir, « cage ») : l'ekphrasis semble donc quasi-impossible à mener. C'est qu'il s'agit pour l'auteur d'insister sur la volonté des

94 Voir notamment le tableau d'Henry de Groux, Zola aux outrages, 1898, Médan, Musée Zola. À ce sujet, se reporter à Tillier, Les artistes... 225 et 233. 
instances politiques de placer Picquart hors du monde, hors de la vue, ce que corrobore la reprise des mêmes termes à la toute fin du texte, en un retour très bref de la description : " Je vois, derrière le grillage, sa main qui m'envoie un affectueux bonjour... Et son visage s'estompe, s'efface, comme s'il s'enfonçait dans le brouillard... » (244). En insistant sur le phénomène qui conduit à distinguer Picquart, le critique donne à voir le processus qui mène à la vérité. La persévérance qui accompagne la difficulté à voir, et qu'induit cette recherche de la vérité jusque dans le cachot, suggère l'idée de discernement, laquelle est, on le sait, au cœur de la démarche critique, comme l'atteste l'étymologie (la signification du verbe grec krinein est « discerner », " trier »). En outre, on peut constater que la description semble aussi être le fait d'un Mirbeau critique d'art : bien qu'ici l'impression s'avère " pénible », on songe aux nombreux écrits que celui-ci a consacrés à Monet et aux impressionnistes qui furent ainsi l'objet des autres combats de l'écrivain, des " combats esthétiques ». Mais l'auteur quitte bientôt le lexique impressionniste pour recourir à celui de la netteté :

En réalité, à de certaines heures, ou par les ciels bas, les jours crasseux, comme hier, on ne distingue que les deux yeux, deux yeux clairs, limpides, heureux et qui vous font un accueil amical.

Nous causons. Et c'est pendant la demi-heure réglementaire, un grand plaisir et un grand charme.... De même que son regard a conservé sa vivacité, de même sa voix a gardé son timbre net. Aucune altération. Pas la moindre fatigue et la moindre nervosité. À mesure qu'il me parle, et mes yeux s'habituant à la pénombre, je le vois mieux, je le vois tout à fait. Il est le même qu'il y a six mois, un peu plus pâle seulement, non d'une pâleur souffrante, mais de cette pâleur spéciale qu'ont les personnes qui ne sortent jamais... (242-243)

Ce passage conduit à la notion d'enargeia. En effet les rhéteurs antiques voyaient en elle " la faculté d'atteindre à la transparence du signe », elle était ainsi « soumise à un critère d'utilité : elle doit procurer une re-présentation crédible d'éléments attenant à la cause, apte à émouvoir le juge [...]» (GalandHallyn 100). Perrine Galand-Hallyn évoque également la dimension « exhibitionniste » de l'enargeia littéraire. En effet, " la théorie même de la "vive représentation" prévoit sa propre dénonciation en tant qu'artifice, puisque l'auteur [...] doit intervenir dans son texte, établir un lien personnel avec le sujet traité. Le plus souvent, cette intervention prend la forme d'un éloge ou d'un blâme portés par le descripteur sur l'objet décrit » (101). Dans cet extrait de la préface on notera le terme "vivacité » associé à celui de « regard » comme si l'enargeia, propriété de l'ekphrasis, était désignée. En outre, Mirbeau insiste sur l'intégrité du colonel en soulignant la constance de cette vivacité. La description du personnage, dont les traits physiques n'ont pas changé, permet de soutenir son intégrité morale et 
ce geste est parallèlement accompagné de la vision de plus en plus nette de l'écrivain ${ }^{95}$. Ainsi, tout au long de ce morceau, la dénonciation de l'inversion des principes républicains est d'autant plus rendue évidente que le colonel reste le même, en d'autres termes il incarne la vérité inaltérable. Ce trait est corroboré dans le portrait en homme de lettres fin et érudit qui est donné de Picquart dans le passage qui suit, troisième et dernier moment de la préface.

Si le morceau d'ekphrasis est bien identifiable, la notion d'évidence adossée à celle de justice n'en traverse pas moins l'ensemble de ce plaidoyer dont la nature hybride l'apparente davantage encore au genre de l'essai. La préface de Mirbeau peut ainsi autant être considérée comme texte autonome, contenant une ekphrasis, que dépendant des lithographies. Elle prend même valeur d'allégorie, ${ }^{96}$ cette figure qui est volontiers associée « à la recherche d'une enargeia, d'une evidentia destinée à donner au discours toute sa force » (Garde Tamine, «Avant-Propos » 5), comme en témoigne la définition de Fontanier : «Elle consiste dans une proposition à double sens, à sens littéral et à sens spirituel tout ensemble, par laquelle on présente une pensée sous l'image d'une autre pensée, propre à la rendre plus sensible et plus frappante que si elle était présentée directement et sans aucune espèce de voile » (114). Quelle serait cette pensée ? Ne s'agit-il pas de porter un regard sur les arts, y compris celui de l'avant-garde, comme porteurs de vérité, bien loin de leur disqualification par la réduction classique au domaine de l'illusion trompeuse, bien loin aussi de l'art pour l'art et des artistes peu acquis à la cause dreyfusarde ? Ne s'agit-il pas pour Mirbeau, d'obédience anarchiste, à présent séduit par la pensée socialiste, après avoir résolument tourné le dos à l'antisémitisme, ${ }^{97} \mathrm{~d}$ 'affirmer le parti des intellectuels

95 On pourrait recourir à d'autres notions de la rhétorique. Juliette Dross a par exemple analysé « à l'époque romaine [l'] établissement d'une relation entre l'enargeia et l'imagination de l'orateur, ou phantasia ». L'auteur souligne le rapprochement entre evidentia et enargeia.

96 Joëlle Gardes Tamine et Marie-Antoinette Pellizza explicitent ainsi l’allégorie, à laquelle elles donnent une " définition restreinte », puisqu'elle prend appui sur le double-sens plutôt que sur la figuration. Lallégorie est une " énigme, qui demande à être résolue par celui qui l’a perçue» (25). Cette énigme, autrement dit le deuxième sens du « double-sens » propre à l'allégorie, peut s’adosser à " une vérité philosophique ou religieuse enfouie sous le sens littéral », tout en ayant la particularité, à la différence de la métaphore, de « repose[r] sur l’absence rigoureuse de contact entre le littéral et le figuré » (28). Sur la place de l'allégorie dans l’art du second XIX siècle, voir aussi Vouilloux 2006.

97 Sur les positions politiques de Mirbeau, on peut consulter les articles « Anarchie », « Antisémitisme », « Socialisme », dans le dictionnaire en ligne de la Société Octave Mirbeau. L'écrivain explique son abandon des positions antisémites dans les articles suivants : «Palinodies! » (L'Aurore, 15 novembre 1898), « Un mot personnel» (L'Aurore, 21 décembre 1898); repris dans Mirbeau 159-164 et 193-194. Voir aussi les commentaires de Michel et Nivet sur l'antisémitisme qui a cours à cette époque (75 et 193) et ceux de Maurice Agulhon qui met en évidence la complexité de l'« antisémitisme nouveau » issu de « la convergence d'hostilités hétérogènes » (La République I, 122-123). 
dreyfusards auquel sont associés les écrivains et les artistes ? Dès lors l'évidence de l'enargeia côtoie le double sens de l'allégorie d'où résulte la force de ce texte. La préface assure donc bien son rôle, celui d'orienter la lecture et de préfigurer les éléments à venir : l'enargeia annonce la lumière, objet de figuration et de représentation dans les lithographies de l'album qui s'attachent elles aussi à la question de la justice tout en ayant recours essentiellement à l'allégorie, sous son aspect cette fois iconographique.

\section{«Hommage des artistes à Picquart» : l'évidence par la lumière}

L'Affaire Dreyfus est caractérisée par la multiplication des publications et le développement d'une imagerie qui résulte de l'opposition violente entre les dreyfusards et les antidreyfusards. Comme l'a analysé Bertrand Tillier, l'imagerie politique est souvent dénigrée en raison du caractère éphémère des publications et de leur manque de qualité. Les artistes impliqués dans l'Affaire manifestent une volonté d'infléchissement de ce discrédit (Les artistes... 313-314). Le portfolio Hommage des artistes à Picquart s'inscrit dans ce renouvellement de l'imagerie politique par les trois parties qui la constituent et par la qualité esthétique visée par les artistes qui permet d'enter leur œuvre sur l'histoire en dehors de l'affiche politique de circonstance..$^{98}$ En outre sa cohérence tient au fil de l'évidence qui s'y déploie. Qu'elle soutienne ou non une allégorie, celle de la Vérité, la figuration de la lumière prime dans les douze lithographies, soulignant l'évidence de l'innocence du colonel Picquart, et partant, celle de Dreyfus. Neuf estampes sur douze font particulièrement appel aux ressources multiples offertes par la lithographie en noir et blanc (travail du trait et de la ligne, gamme des gris, jeu sur les contrastes) pour souligner plastiquement le rôle de la vérité au fondement de la justice ${ }^{99}$. Ces artistes inscrivent d'emblée leur œuvre dans la pensée dreyfusarde, où abondent les références à la vérité.

Un premier ensemble de planches se distingue dans lequel les artistes ont recours au registre

98 Dans son numéro du 16 février 1899 le journal L’Aurore souligne l'originalité et l'importance de l'album : «Cette publication, d’un goût artistique très élevé, contient, en outre de douze lithographies signées : Anquetin, Cornillier, Gumery, Hermann-Paul, Maximilien Luce, Manzana, Perroudon, H. Petitjean, Rault, Rysselberghe, Sunyer et Vallotton, une préface d'Octave Mirbeau, une notice de Henri Raynaldi et les listes complètes des amis de la Vérité qui se sont empressés de signer les protestations en faveur de la mise en liberté de Georges Picquart. Cet album restera comme un des documents les plus curieux et les plus importants de l'affaire qui passionne l'opinion publique depuis plus d'un an ; c’est une page d'histoire » (cité dans Mirbeau 239).

99 On ne mentionnera donc pas les estampes d'Anquetin, Gumery et Perroudon. La planche de Gumery met plutôt en avant l'allégorie de la Gloire, voir Tillier 234. 


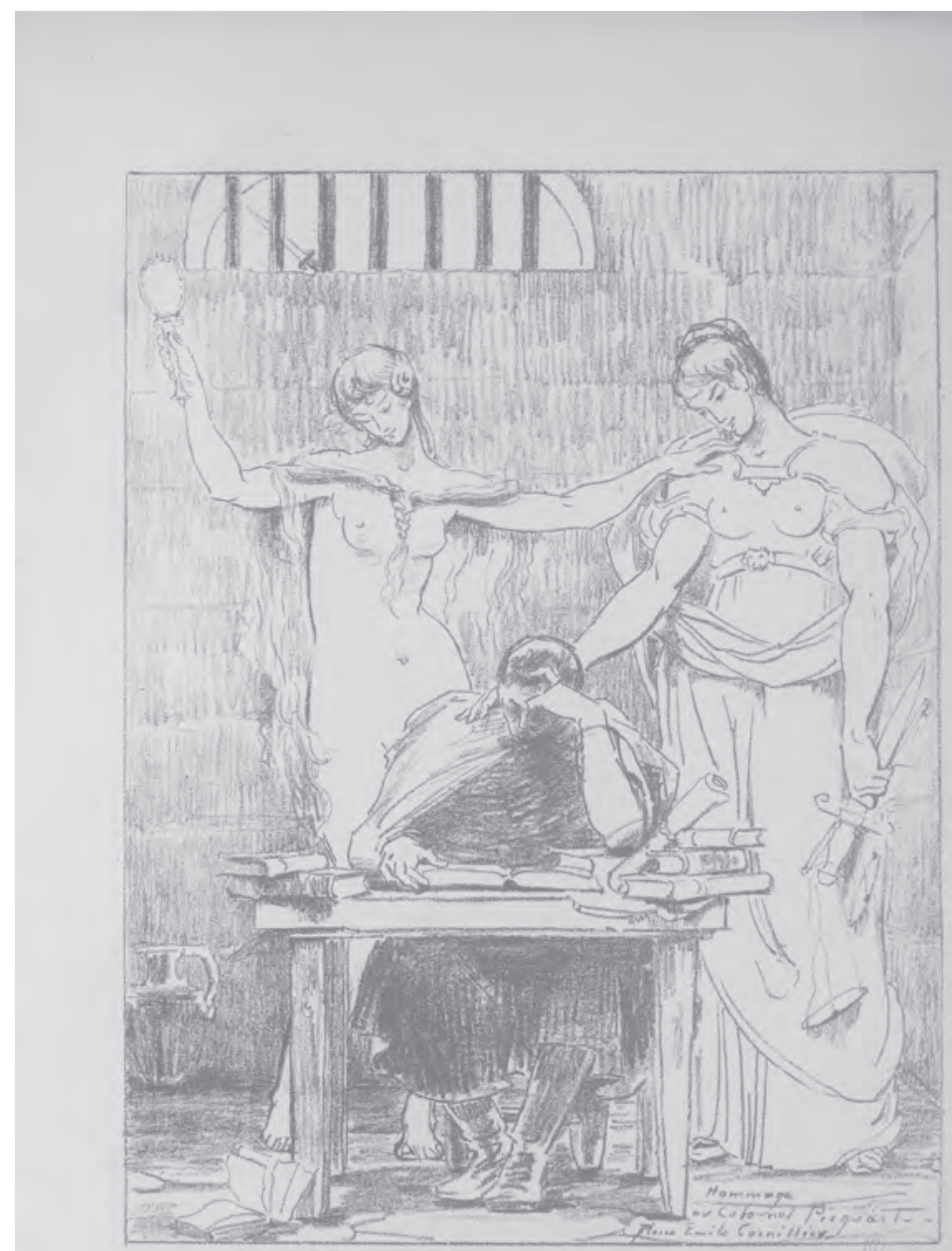

Fig. 1. Pierre-Émile Cornillier (1862-1948), lithographie, Hommage des artistes à Picquart, 1899. Paris : Société libre des gens de lettres, Paul Brunet et Félix Thureau (ed.), $40,5 \times 31 \mathrm{~cm}, \mathrm{n}^{\circ} 200$ (Fol K 35). Paris : Bibliothèque de l'Institut National d'Histoire de l'Art. 
Interfaces 42 (2019-2020)

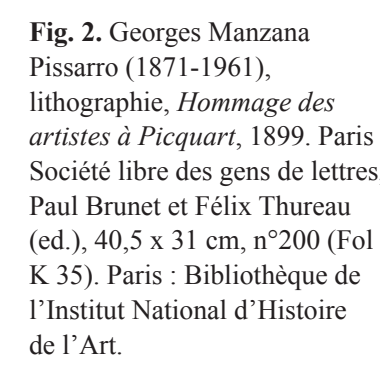

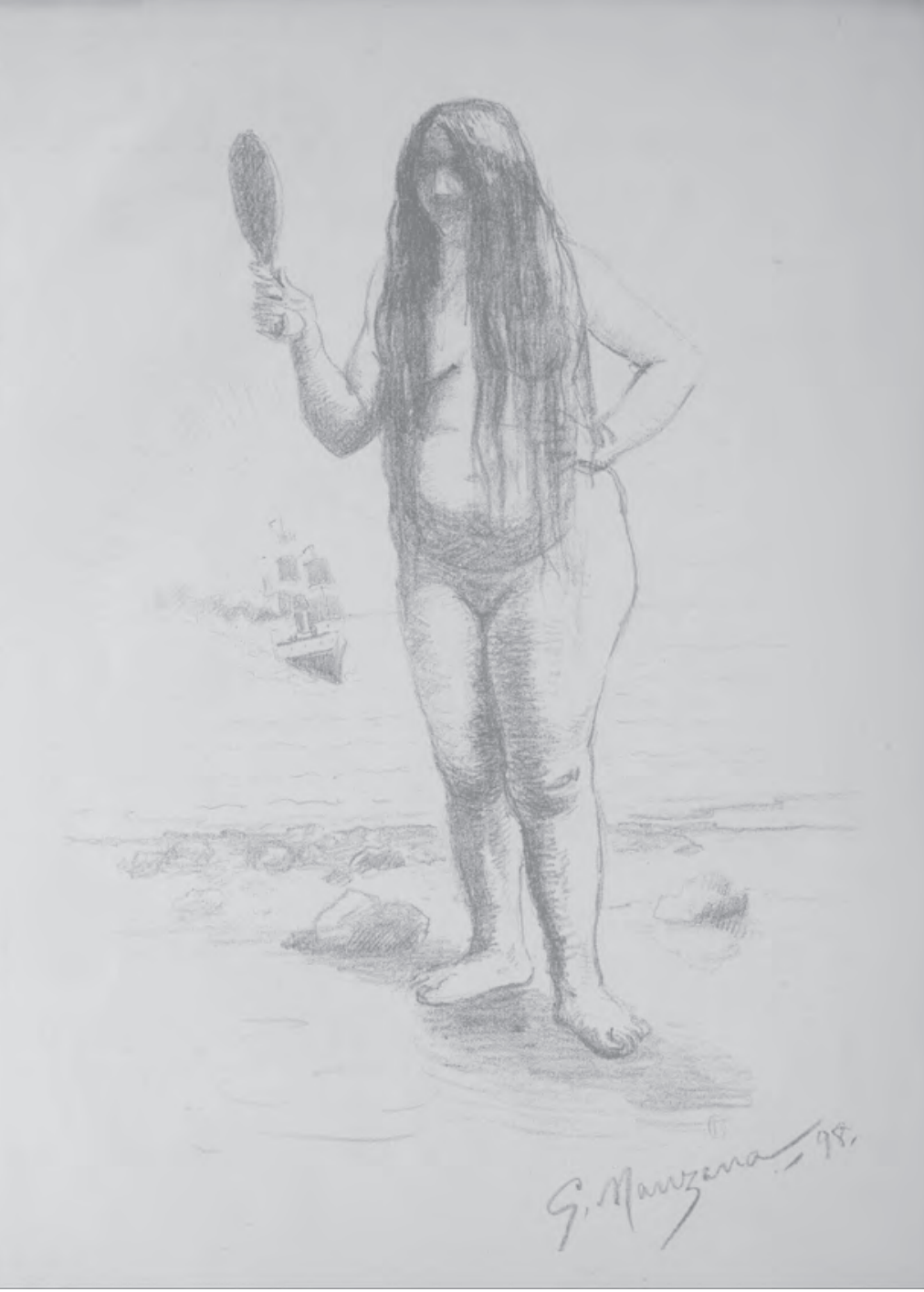




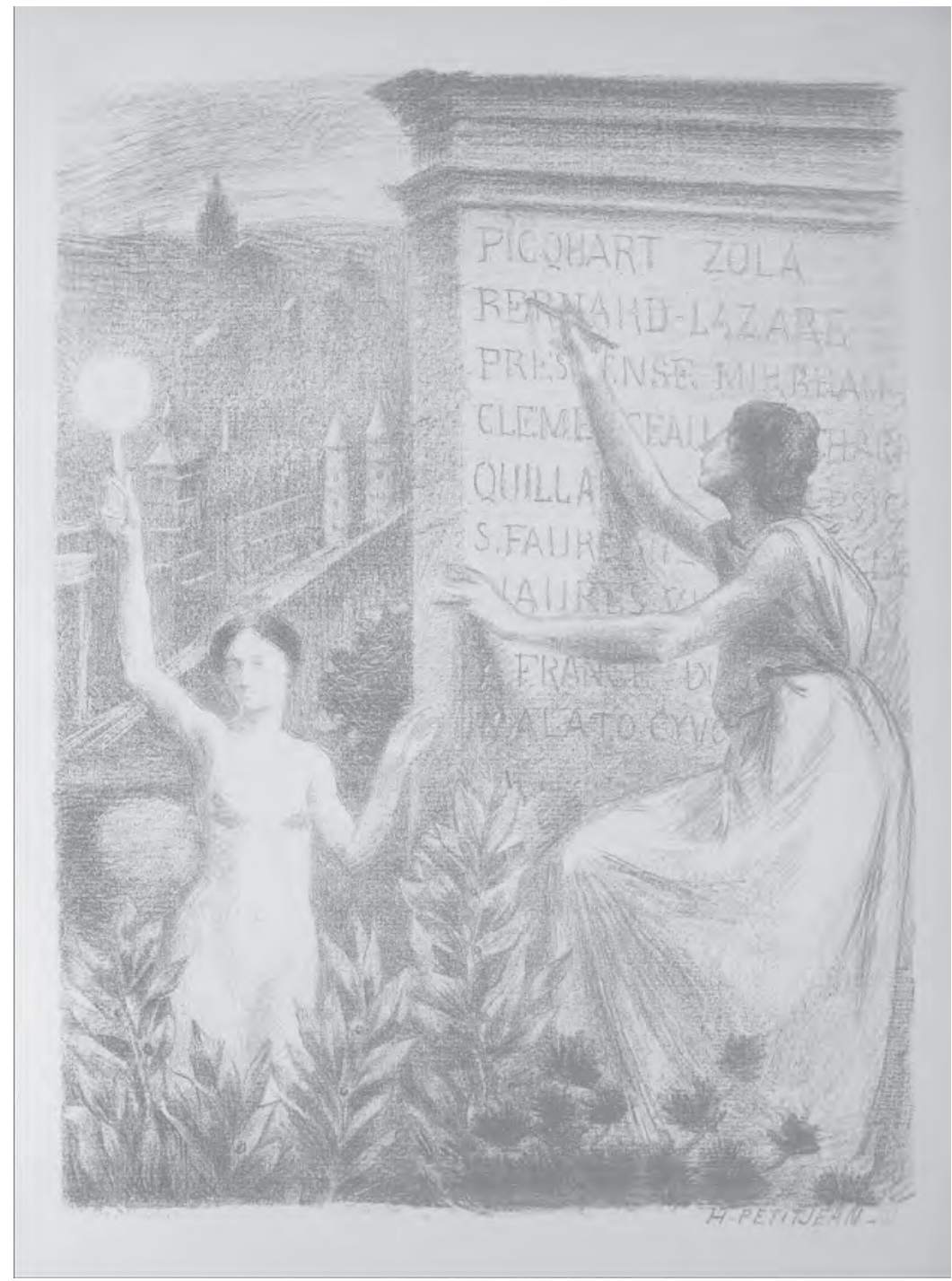

Fig. 3. Hippolyte Petitjean (1854-1929), lithographie, Hommage des artistes à Picquart, 1899. Paris : Société libre des gens de lettres, Paul Brunet et Félix Thureau (ed.), $40,5 \times 31 \mathrm{~cm}, \mathrm{n}^{\circ} 200$ (Fol K 35). Paris : Bibliothèque de l'Institut National d'Histoire de l'Art. 
de la peinture d'histoire par la présence de l'allégorie de la Vérité. Celle-ci participe de la construction de l'imaginaire républicain, celle des institutions et des " Grands hommes », sous la III République. Comme l'a bien montré Bertrand Tillier, « quand l'affaire Dreyfus raviva cette figure, [l'allégorie] appartenait [...] surtout au vocabulaire de l'art académique - comme l'indiquent les tableaux de Jules Lefebvre ou de Jean-Léon Gérôme - et de la statuaire publique de la Troisième République » (Les artistes... 240). Elle tient une place essentielle dans les textes produits par Zola, de sorte qu'elle viendra particulièrement nourrir le combat des intellectuels lors de l'affaire Dreyfus. Que l'on songe à la formule qui clôt l'article consacré au sénateur dreyfusard Auguste Scheurer-Kestner : « La vérité est en marche, et rien ne l'arrêtera » (77), reprise quelques semaines plus tard, en janvier 1898, dans la célèbre « Lettre à M. Félix Faure, Président de la République » (139). Dans l'iconographie traditionnelle l'allégorie de la Vérité est incarnée par une femme nue «pour montrer que la naïveté lui est naturelle, et qu'elle n'a pas besoin d'explication pour se faire entendre », selon Cesare Ripa, qui, dans son Iconologie lui attribue un livre ouvert, une branche de palme, et « un soleil qu'elle regarde [...] pour montrer aussi combien elle chérit la clarté » (295), l'astre étant source de Vérité. Mais l'iconographie traditionnelle substitue également le miroir, attribut de la Prudence, de la Sagesse, au soleil. La Vérité est en effet associée à une source lumineuse. Avec Dame Justice, elle accompagne Picquart dans son cachot (Pierre-Émile Cornillier, fig. 1) lequel, entouré de livres, est figuré en intellectuel concentré, plutôt qu'en prisonnier abattu. Notons que Dame Justice, dont l'attribut principal est la balance, et que l'on trouve sur les murs de nombre de tribunaux (Curtis et Resnik) n'est représentée qu'une seule fois dans l'album, comme si l'institution républicaine était décrédibilisée. Dans le dessin de Georges Manzana Pissarro (fig. 2) la Vérité nue est loin de correspondre à une femme idéalisée. Le visage occulté par les ombres, elle se regarde dans un miroir mais aucune lumière n'en émane. C'est que la situation est loin d'être glorifiée : le bateau qui s'apprête à aborder évoque celui qui viendra chercher Picquart pour l'amener en Tunisie ${ }^{100}$ afin de l'empêcher de poursuivre ses déclarations. Tillier voit dans ce dessin une dénonciation de circonstance : s'attaquant aux antidreyfusards qui s'approprient les valeurs de Justice et de Vérité, Manzana présenterait ainsi « la Vérité hideuse et répugnante vue avec les yeux des antidreyfusards » (Les artistes... 249). A contrario, le soleil que l'allégorie de la Vérité tient de sa main droite produit une intense lumière qui éclaire le monument sur lequel une allégorie de l'Histoire inscrit les noms des intellectuels dreyfusards (Piquart, Zola, Clemenceau, Jaurès...), autrement dit ceux dont la postérité se souviendra (Hippolyte Petitjean, fig. 3). Ils prennent ainsi le statut de « héros intellectuels » selon la formule de Bertrand Tillier (Les artistes... 232) qui souligne ainsi l'alliance

100 Picquart est affecté dans le sud de la Tunisie le 6 janvier 1897 pour rejoindre le $4^{\mathrm{e}}$ régiment de tirailleurs (Agulhon 147 et 192). 


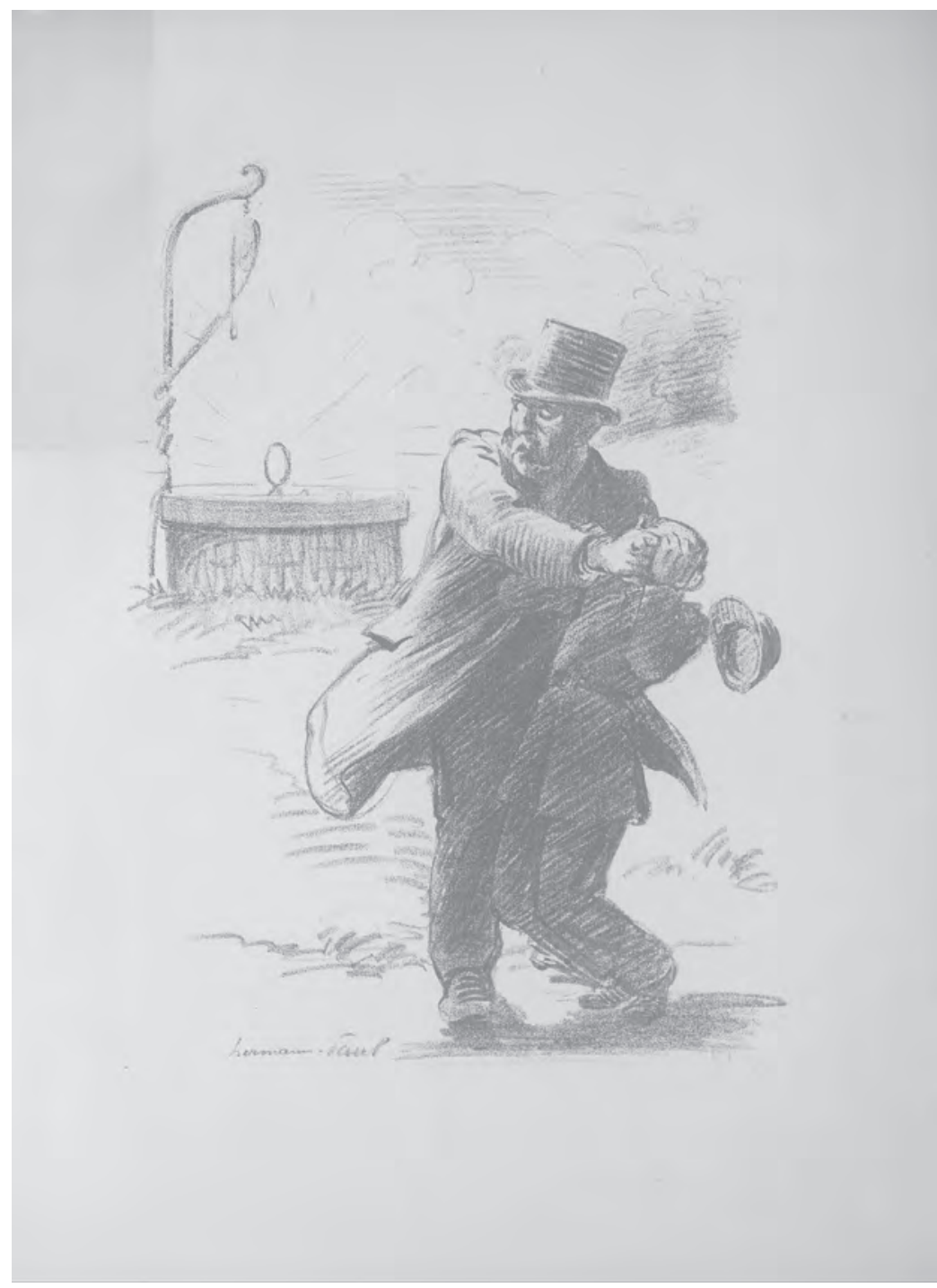

Fig. 4. René Georges HermannPaul (1864-1940), lithographie, Hommage des artistes à Picquart, 1899. Paris : Société libre des gens de lettres, Paul Brunet et Félix Thureau (ed.), $40,5 \times 31 \mathrm{~cm}, \mathrm{n}^{\circ} 200$ (Fol K 35). Paris : Bibliothèque de l'Institut National d'Histoire de l'Art. 
Fig. 5. Theo Van Rysselberghe (1862-1926), lithographie, Hommage des artistes à Picquart, 1899. Paris : Société libre des gens de lettres, Paul Brunet et Félix Thureau (ed.), $40,5 \times 31 \mathrm{~cm}, \mathrm{n}^{\circ} 200$ (Fol K 35). Paris : Bibliothèque de l'Institut National d'Histoire de 1'Art.

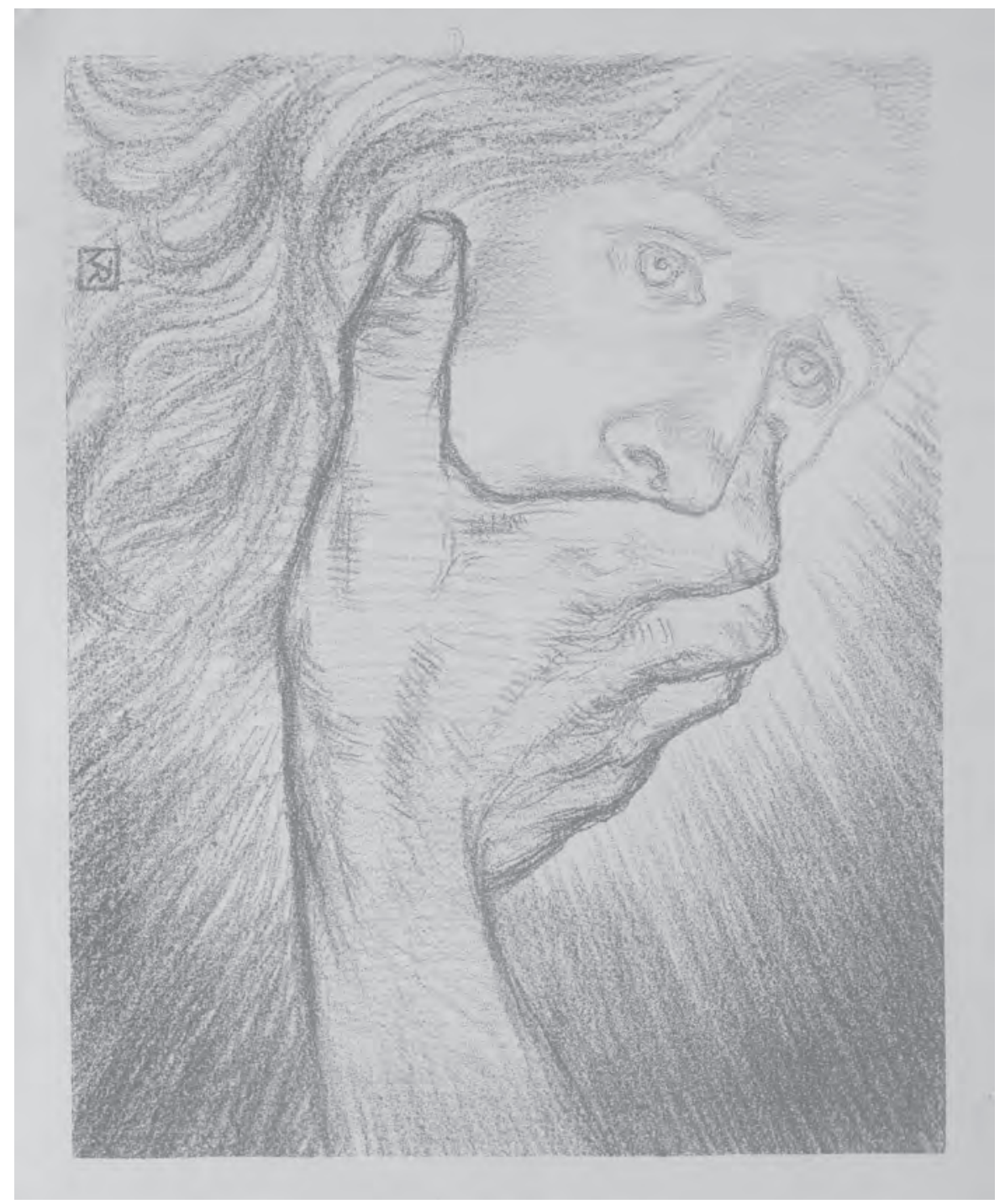



inédite de «l'action [à] la réflexion ». Dans l'estampe d'Hermann-Paul le réalisme semble vouloir primer l'allégorie. En effet, la Vérité n'est représentée qu'en partie, de manière métonymique : seul le miroir dépasse du puits ${ }^{101}$ (fig. 4). À la différence de cette planche, dans laquelle se détache la silhouette sombre et imposante du Président Félix Faure qui bâillonne Picquart (le motif de la main est repris dans le dessin de Théo van Rysselberghe figurant de manière énergique, par le dégradé, la Vérité muselée fig. 5), c'est la blancheur, la Vérité désincarnée, qui est mise en relief dans la lithographie de Joachim Sunyer (fig. 6). Isolée dans le dessin où domine le noir, la réserve permet l'identification de cette forme fantomatique et lumineuse qui se dresse au sein d'une foule hurlante hostile. La scène s'apparente à un bûcher qui symbolise le monde inversé dénoncé par Mirbeau : la Vérité est sacrifiée. Ce premier ensemble montre la place originale que tient l'allégorie de la Vérité, entre conventions académiques et inscription dans l'actualité. À la faveur des lignes simples du dessin, mais aussi du traitement des gris, des contrastes appuyés entre le noir et le blanc, l'allégorie vient affirmer l'écart entre la réalité et la vérité.

Le deuxième ensemble de lithographies (Louis Rault, Maximilien Luce, Félix Vallotton) explore lui aussi la figuration de la vérité via la lumière et les ressources du noir et du blanc mais la médiation proprement allégorique ne prime pas. On s'attachera particulièrement à la planche de Vallotton (fig. 7) qui clôt l'album : le domaine du symbole et de l'intimité prévaut sur celui de l'allégorie et de l'histoire. La répartition expressive du noir et du blanc, la simplicité du graphisme qui synthétise formes et objets y contribuent. La scène est éclairée par une simple bougie plutôt que par un attribut allégorique. Le rideau qui borde le lit d'une part, et les ombres portées formées par les aplats de noir d'autre part, permettent à Vallotton de théâtraliser le moment qui conduit le président du Conseil Jules Méline (Kleeblatt 218), antidreyfusard, à une révélation qui le fait sortir de son sommeil : l'innocence de Dreyfus, clamée par Picquart. Vallotton crée une mise en abyme dans le recueil de planches : il emboîte le procès de Dreyfus (objet de son dessin) dans celui de Picquart (objet de l'album), donnant ainsi relief et profondeur à l'Affaire. L'inscription « il est innocent » semble flotter sur la surface du dessin, c'est-à-dire hors de l'espace iconique, ce qui lui confère une valeur de vérité générale. Tournée vers le personnage, autrement dit renversée, l'inscription correspond en outre autant au discours énoncé (ou plutôt au cri proféré) par Picquart qu'au discours intérieur de Méline. La gravité de la révélation est accentuée par l'attitude du personnage traduite par les lignes : buste redressé, bouche ouverte,

101 Le motif du puits qui prend sa source dans l'adage « la vérité sort du puits », attribué à Démocrite, est très présent dans l'iconographie de l'art de la fin du XIX ${ }^{e}$ siècle et de l'affaire Dreyfus (voir notamment le tableau de Jean-Léon Gérôme, La Vérité sortant du puits, 1896, huile sur toile, Moulins, musée Anne-de-Beaujeu). À ce sujet voir le catalogue d’exposition La vérité nue : enquête autour d’une allégorie et Tillier, Les artistes... 240-251. 
yeux écarquillés, bras en avant. Elle prend une valeur plus générale encore. " Il est innocent » : cette formule est en effet polyphonique, elle résonne entre autres avec le titre d'une célèbre affiche de 1898 (« Dreyfus est innocent») ou encore avec le titre d'une lithographie (« Histoire d'un innocent »), lesquelles ont leur pendant antidreyfusard où prime le mot « traître $»{ }^{102}$ Cette formule résume à elle seule l'ensemble du discours dreyfusard. Mirbeau saura s'en souvenir en la faisant proférer par le personnage du poète au dîner de l'« Illustre écrivain $»{ }^{103}$

L'enchaînement des lithographies engage ainsi un passage de l'allégorie au symbole, autrement dit un passage des traits spécifiques à la peinture d'histoire à ceux de l'imagerie plus populaire, liée au dessin de presse et à l'illustration, propre au XIX ${ }^{\mathrm{e}}$ siècle. Cette distinction accompagne d'un côté l'héroïsation de Picquart, et de l'autre le parti de l'homme, citoyen d'une République défaillante. En outre, le symbole de la lumière sert la notion d'évidence puisque l'une des manières de distinguer les deux notions consiste à dire que l'allégorie demande davantage d'analyse que le symbole qui frappe d'emblée. Enfin notons que le ton de la gravité domine ces compositions, c'est pourquoi l'album emprunte peu au registre satirique et ne recourt pas à la caricature : il se hisse au-delà du combat dreyfusards-antidreyfusards, il tourne le dos à l'imagerie violente des antidreyfusards (celle par exemple de Bob et de Lenepveu).

Dans cet album, le principe d'évidence est amplifié par un troisième élément : la liste des protestataires. Se pose dès lors la question de la portée politique de cet album et plus précisément de l’idée républicaine qui y est défendue.

102 Affiches, Anonyme, " Histoire d'un innocent ", 1898, " Histoire d'un traître ", 1899 ; affiches avec photographies, « Dreyfus est un traître », « Dreyfus est innocent », E. Charaire imprimeur, 1898. Voir Kleeblatt, 210-211 et 194-195.

103 «Que peuvent tous les jugements et toutes les sentences d'un conseil de guerre contre cette impression mystérieuse et révélatrice qui me pousse à crier : "il est innocent ! Il est innocent !" [...] L'Illustre Écrivain écumait. Il imposa le silence : — Et quand même Dreyfus serait innocent ? vociféra-t-il... il faudrait qu'il fût coupable quand même... il faudrait qu'il expiât, toujours... même le crime d'un autre... C'est une question de vie ou de mort pour la société et pour les admirables institutions qui nous régissent !.. La société ne peut pas se tromper... Les conseils de guerre ne peuvent pas se tromper... L'innocence de Dreyfus serait la fin de tout!" , Octave Mirbeau, «Chez l'Illustre écrivain », Le Journal, 28 novembre 1897 (48-49). Michel et Nivet précisent que « cette opinion sera formulée, entre autres, par Barrès » (49). 
Fig. 7. Félix Vallotton (18651925), lithographie, Hommage des artistes à Picquart, 1899. Paris : Société libre des gens de lettres, Paul Brunet et Félix Thureau (ed.), 40,5 x $31 \mathrm{~cm}$, $\mathrm{n}^{\circ} 200$ (Fol K 35). Paris :

Bibliothèque de l'Institut National d'Histoire de l'Art.

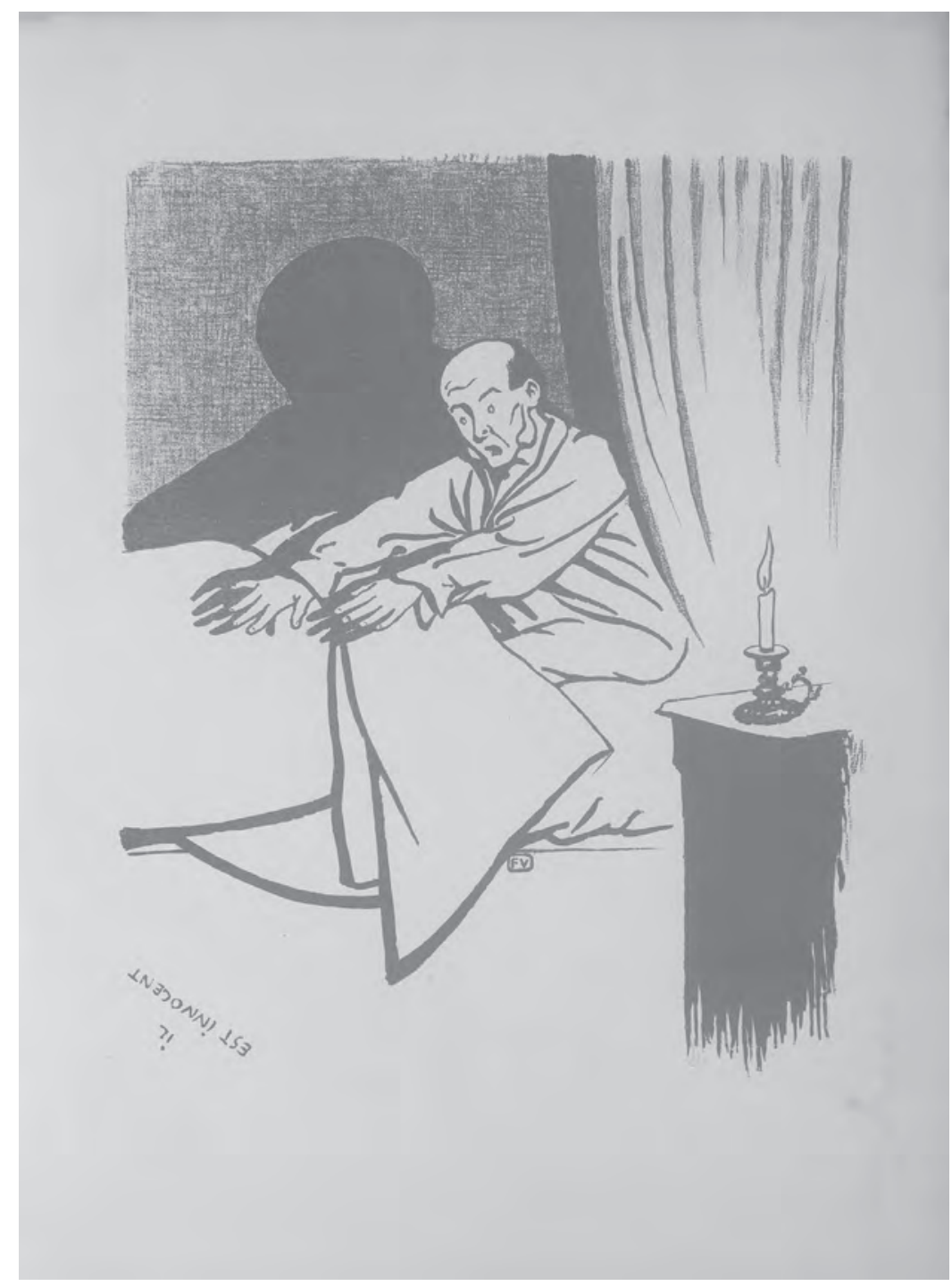




\section{L'album et la liste de protestataires : quelle idée de la République ?}

La mise en avant de l'évidence, on l'a vu, participe de la défense de la justice pour Mirbeau, celle-ci visant la vérité sur laquelle les artistes de l'album insistent quant à eux davantage, les principes de justice et de vérité étant aux fondements du discours dreyfusard. Le texte de Mirbeau constitue également une préface à la liste de protestataires dont une partie a d'abord paru dans le Journal L'Aurore. Avec cette troisième section, la publication s'impose dès lors comme un album républicain en faveur de la justice. Mais cette affirmation ne se fait pas sous le mode de l'idéalisation et ne prend pas les atours de l'allégorisation. Le recours à la protestation met en évidence de manière concrète, par l'entremise de la forme démocratique que représente la pétition, les manquements de la République. Comme le rappelle Vincent Duclert l'affaire Dreyfus révèle « une justice qui s'oppose au droit et rejette le principe de vérité » (Duclert, « La Justice » 77). L'album et la protestation répondent en cela à un moment de crise de la République en tant qu'État de droit. ${ }^{104}$

La justice est l'un des fondements au centre de l'universalisme républicain hérité des Lumières. Pour les tenants de « la tradition républicaine » il incombe à ce régime politique « libéral, démocratique, laïque et parlementaire », qui s'appuie sur les valeurs de fraternité et d'humanité, de participer à la réalisation du Bien. C'est pourquoi « il allait de soi que la justice réclamée par Dreyfus fût un combat "républicain" » (Agulhon, « La République » 54). L'Affaire Dreyfus met au jour les oppositions politiques entre gauche et droite. Comme l'a rappelé Maurice Agulhon :

On commence à appeler « révisionnistes », en attendant de dire " dreyfusistes » ou « dreyfusards », ceux qui, arguant de la justice, veulent réviser le procès, parce qu'il fut mal conduit, inique, ou simplement douteux. Leur raisonnement implique que la justice, l'échelle des valeurs reconnue comme bonne par la morale universelle, bref la morale, ait quelque chose à voir avec la politique - ce qui est sans doute la plus permanente et la plus essentielle des définitions de la gauche. (La République I 147)

104 « À la fin des années 1880, l'optimisme républicain est donc remis en cause à la fois par les faits (les scandales financiers, les affrontements sociaux croissants, l'instabilité politique liée aux divisions entre républicains modérés et radicaux, la dépression économique) et par de nouvelles idéologies d'inspiration évolutionniste aux conclusions opposées : pour le darwinisme social, l'évolution aboutit au triomphe des plus forts ; pour le socialisme, les plus faibles ne peuvent l'emporter qu'en refusant collectivement l'ordre établi. Pour les uns comme pour les autres, l'élitisme républicain est donc un mensonge, et l'élite républicaine est assimilée par les antisémites et les socialistes à une féodalité financière (à cause des scandales) qui développe un nouveau favoritisme peu différent de celui des orléanistes (référence est faite aux épurations massives des débuts du régime) », Charle (74). 
Du côté des antidreyfusards nationalistes tel Barrès, ceux qui n'envisagent pas de réviser le procès de Dreyfus, le pays prime l'universalisme abstrait, comme l'explique l'historien, spécialiste de la République :

Le raisonnement antirévisionniste est donc, à son tour, exemplaire de la pensée de droite par ces deux aspects conjoints et solidaires : accepter un non-universel, la France, comme sommet de la hiérarchie des valeurs ; et affirmer la spécificité du politique et de ses règles d'action au lieu de soumettre l'action politique aux règles de la morale générale. (La République I 148)

L'auteur du Siècle des intellectuels insiste quant à lui sur la manière résolument opposée de considérer les relations entre morale, principes et politique : «Vérité ? Justice ? Abstractions... Vérité ? Justice ? Là est justement la question pour Émile Zola » (Winock 19).

Cette question sera partagée par d'autres intellectuels mais pas seulement, comme en témoigne la liste prodigieuse de protestataires qui constitue la troisième partie de l'album. La protestation répond ainsi à l'injustice. Durant l'Affaire, elle sera une forme prisée par les deux camps, et c'est par son biais que se forge le parti des intellectuels. S'il s'agit d'abord de se constituer contre l'élite, les « intellectuels », issus de l'avant-garde " qui se définit par opposition à la littérature de consommation » (Charle 113), en viennent ensuite à intégrer l'élite dans leur regroupement. Cette liste constitue un témoignage particulièrement fort car avec elle une forme nouvelle émerge : les signataires embrassent en effet un large spectre de professions et de classes sociales qui ne sont pas organisées selon une hiérarchie à la manière de la pétition patriotique, c'est-à-dire antidreyfusarde, de la Ligue des Patriotes. On y trouve par exemple le nom d'un garçon boucher, non loin de la mention d'un groupe, les employés de l'Huilerie Provençale, représentés par le directeur de la fabrique, lequel groupe côtoie le nom de Victor Basch, célèbre professeur de la faculté de Lettres (Brunet et Thureau 56-57). Se détachent également les noms des peintres Claude Monet et Édouard Vuillard (Kleebatt 221). Comme le précise Christophe Charle, "dans cette démocratie en listes », "les universitaires, si prééminents dans les premières protestations, sont à présent noyés dans la masse » (148). Les deux aspects de la pétition, selon qu'on l'envisage du point de vue quantitatif ou qualitatif, sont associés. L'auteur de Naissance des intellectuels a souligné les enjeux de cette « arme politique » :

[...] l'affaire Dreyfus renoue avec la tradition des pétitions de masse pour faire contrepoids au pouvoir politique impuissant et substitut à l'opinion publique passive. Les pétitions réalisent ainsi l'alliance insolite des gens de qualité et des gens quelconques, ce qui est apparu aux contemporains comme révolutionnaire et un peu scandaleux puisque étaient ainsi mises 
en œuvre deux stratégies opposées : une stratégie élitiste où le poids compte plus que le nombre, et une stratégie démocratique ou les gros bataillons sont un argument dans la lutte pour la détention de la vérité politique. Or, dans l'imaginaire politique du temps [...] ces oppositions entre les intellectuels et le reste de la société ou entre l'élite et la foule paraissaient indépassables. (142)

Au sein des gauches, d'aucuns ont pu estimer que l'Affaire, n'impliquant que des bourgeois, ne méritait pas qu'on s'y investisse, Dreyfus n'étant « qu'un ennemi de classe parce qu'il était officier, qu'il était riche, et qu'il était juif » (Duclert, Jaurès... 36). La liste de signataires semble donc en partie déjouer ces clivages et oppositions. Immense et massive, autoconstituée autour d'une même cause, ce que « l'effet de clôture » qui découle de l'ordre chronologique et alphabétique — autrement dit « un principe de distribution arbitraire mais fixe » (Hamon 26) - accentue, cette liste se présente d'elle-même, elle énonce l'évidence d'une injustice et la volonté de justice, en instituant le passage de l'opinion individuelle à la « coalition d'opinions collectives » (Charle 140). C'est pourquoi on peut voir dans cette pétition l'expression du principe démocratique.

Mais le lien entre démocratie et république, c'est-à-dire la représentation politique, est aussi présent si l'on se réfère au jury de la cour d'assise qui participe du pouvoir législatif. Pour réaliser les principes républicains de justice et de vérité les jurés constituent en effet un maillon indispensable dans l'institution républicaine. Frédéric Chauvaud rappelle que le jury « est perçu comme le droit donné au peuple de juger. Dans l'affirmation des principes, il est le cadre du civisme, judiciaire et républicain, et l'incarnation de la citoyenneté » (199). Par sa forme même, la liste "Les Noms » désigne la liberté d'expression, soubassement de la démocratie républicaine, et partant, elle incarne la République par ses manquements, puisque « l'affaire Dreyfus [...] a incarné l'Erreur» (Chauvaud 200). «Les Noms » s'ajoutent à la préface et aux lithographies, de sorte que l'album se présente comme un ensemble singulier dans les productions engagées de l'époque. Les écrivains et les artistes sont situés sur le même plan que les autres citoyens, celui de la politique, davantage que celui du groupe ou de la communauté. Les différents acteurs et énonciateurs de l'album soulignent les manquements de la République mais pour mieux la défendre. La liste des protestataires qui participe de l'album met au premier plan l'exigence de justice tout en s'imposant comme force démocratique. Il s'agit pour les signataires mais aussi pour tous les participants de l'album de viser la vérité au-delà de la simple juxtaposition d'opinions. Elle insiste sur la séparation entre le domaine de la justice et le domaine de la politique, ce que Mirbeau invoque par le biais du jeune poète présent au dîner de «l'illustre écrivain » à qui il répond en ces termes après que celui-ci a défendu l'ordre et la société : « Je vous parle justice !... Et vous me répondez politique !... Vous êtes de pauvres petits imbéciles !.. » (49). 
L'Hommage des artistes à Picquart s'offre ainsi comme un album qui met en jeu démocratie et république via la défense de la vérité et de la justice, « deux exigences constamment réaffirmées par les dreyfusards, qui situent leur combat dans le droit fil de celui des Lumières » comme l'analysent Pierre Michel et Jean-François Nivet (Mirbeau 55). Que ce soit par le texte ou par les lithographies les artistes dénoncent l'injustice criante dont est victime le colonel Picquart et partant, le capitaine Dreyfus. La publication illustre l'un des paradoxes féconds de la modernité esthétique : c'est au moment où les arts s'autonomisent que les collaborations entre artistes et écrivains s'intensifient et se diversifient. Si les moyens employés, propres à chaque médium, sont différents, Mirbeau et les dessinateurs ont en commun de privilégier le recours à une certaine gravité comme s'ils souhaitaient ancrer leur œuvre dans l'histoire. C'est pourquoi dans cet album l'on peut défendre, en reprenant les termes de Bernard Vouilloux («Lire, voir... »), l'idée d'une « co-implication du verbal et du visuel » au service d'une même cause. Avec l'affaire Dreyfus, la III ${ }^{\mathrm{e}}$ République a oublié ses fondements en privilégiant l'esprit de corps, c'est-à-dire en délaissant le droit. La mise en relief de l'enargeia dans la préface de Mirbeau, laquelle dénonce l'inversion des valeurs, a permis de souligner la cohérence de l'album dont les trois parties sont réunies par la mise en avant de l'évidence. Les principes de vérité et de justice sont au centre d'esthétiques très diverses qui ont pour point commun la figuration de la lumière. En outre, la cohérence de l'album découle de la réunion entre les plans esthétique, poétique et politique qu'autorise l'estampe qui « mobilise les deux champs de l'art et de l'édition » (Melot 25) : par la protestation, troisième temps de cet album, les quarante mille citoyens, dont Mirbeau et les douze illustrateurs, affirment la vivacité de la démocratie républicaine.

\section{OUVRages Cités}

AGULHON, Maurice. « La République ». L'Affaire Dreyfus et le tournant du siècle (1894-1910). Ed. Gervereau, Laurent et Prochasson, Christophe. Nanterre : BDIC, 1994. 49-55.

—L La République I, 1880-1932.1990. Paris : Hachette, Pluriel, 2011.

BAT, Marie-Bernard. «Les ekphraseis dans les Combats esthétiques : "L'écriture à l'épreuve de la peinture" ». Cahiers Octave Mirbeau, $\mathrm{n}^{\circ} 21$ (2014) : 108-124.

BRUNET, Paul, et THUREAU, Félix eds. Hommage des artistes à Picquart. Société libre d'édition des gens de lettres, janvier 1899.

CHARLE, Christophe. Naissance des intellectuels (1880-1900). Paris : Éditions de Minuit, 1990.

CHAUVAUD, Frédéric. Article « La Justice». Dictionnaire critique de la République. Ed. Vincent Duclert et 
Christophe Prochasson. Paris : Flammarion, 2002. 196-201.

CURTIS, Dennis et RESNIK, Judith dir. Representing Justice : Invention, Controversy and Rights in City-States and Democratic Courtrooms. New Haven CT, Yale University Press, 2010.

DROSS, Juliette. «Texte, image et imagination : le développement de la rhétorique de l'évidence à Rome ». Pallas 93 (2013) : 269-279.

DUCLERT, Vincent. «La Justice ». L'Affaire Dreyfus et le tournant du siècle (1894-1910). Ed. Gervereau, Laurent et Prochasson, Christophe. Nanterre : BDIC, 1994. 76-90.

_ Jaurès 1859-1914. La politique et la légende. Paris : Éditions Autrement, «Vies parallèles », 2013.

FONTANIER, Pierre. «Allégorie ». Les Figures du discours (1821-1830). Paris : Flammarion, « Champs », 1977. 114. Cité in Vouilloux, Déclins de l'allégorie?. 67.

FLÉJOU, Lucie et POULAIN, Martine. Notice de l'Hommage des artistes à Picquart, site internet de l'Institut National d'Histoire de l'Art. http://blog.bibliotheque.inha.fr/fr/posts/livres-spolies.html

GALAND-HALLYN, Perrine. «L'enargeia, de l'Antiquité à la Renaissance », Les Yeux de l'éloquence. Poétiques humanistes de l'évidence. Orléans : Paradigme, 1995. 99-121

GARDES TAMINE, Joëlle et PELLIZZA, Marie-Antoinette. "Pour une définition restreinte de l'allégorie ». L'Allégorie corps et âme. Entre personnification et double sens. Ed. Joëlle Gardes Tamine. PU de Provence, 2002. 9-29.

GARDES TAMINE, Joëlle. «Avant-propos ». In L'Allégorie corps et âme. Entre personnification et double sens. Ed. Gardes Tamine Joëlle. PU de Provence, 2002. 5-7.

GENETTE, Gérard. Seuils. Paris : Le Seuil, 1987.

GERVEREAU, Laurent et PROCHASSON, Christophe eds. L'Affaire Dreyfus et le tournant du siècle (18941910). Nanterre : BDIC, 1994.

GOYARD-FABRE Simone. Article « République ». Dictionnaire de philosophie politique. Ed. Philippe Raynaud et Stéphane Rials. Paris : PUF, 1996. 657-661.

GRAZIANI, Françoise. « Le miroir de la sagesse ». Miroirs : XVes Entretiens de la Garenne Lemot. Ed. Jackie Pigeaud. Rennes : PU Rennes, 2011. 39-53.

HAMON, Philippe. « La mise en liste. Préambule ». Liste et effet liste en littérature. Ed. Sophie Milcent-Lawson, Michelle Lecolle, Raymond Michel. Paris : Classiques Garnier, 2013. 21-29.

HAYAERT, Valérie. «Allégories de justice ». Imaj-carnets de recherches en Analyse Juridique de l'Image. Billet mis en ligne le 30 juin 2014. http//imaj.hypotheses.org/

HENON Judith, LEYOUDEC Maud dir. La vérité nue : enquête autour d'une allégorie, exposition au musée Anne de Beaujeu, Moulins, du 20 janvier au 29 avril 2012. Moulins : Musée Anne de Beaujeu, 2012. 
HERMOGÈNE. Rhetores graeci, t. VI, Hermogenis Opera. Ed. H. Rabe. Leipzig : Teubner, 1913. Cité dans Vouilloux, Le Tournant « artiste ». 35-36.

KERVÉGAN, Jean-François. Article « Démocratie ». Dictionnaire de philosophie politique. Ed. Philippe Raynaud et Stéphane Rials. Paris : PUF, 1996. 149-155.

KLEEBLATT, Norman L. ed. The Dreyfus Affair. Art, Truth and Justice. The Jewish Museum. Berkeley, Los Angeles, Londres : University of California Press, 1987.

MELOT, Michel. «Introduction ». L'estampe, un art multiple à la portée de tous ?. Ed. Sophie Raux, Nicolas Surlapierre, Dominique Tonneau-Ryckelynck. Lille : PU Septentrion, 2008. 17-25.

MICHEL, Pierre dir. Dictionnaire en ligne de la Société Octave Mirbeau. http://mirbeau.asso.fr/dicomirbeau

MIRBEAU, Octave. L'Affaire Dreyfus. Ed. Pierre Michel et Jean-François Nivet. Paris : librairie Séguier, 1991.

RIPA, Cesare. Iconologie où les principales choses qui peuvent tomber dans la pensée touchant les vices sont représentées. Trad. Baudoin Jean. 1643.

TILLIER, Bertrand. «Les statuts problématiques de l'estampe politique : l'exemple de l'affaire Dreyfus (18981906) ». In L'estampe, un art multiple à la portée de tous?. Ed. Sophie Raux, Nicolas Surlapierre, Dominique Tonneau-Ryckelynck. Lille : PU Septentrion, 2008. 315-321.

— Les artistes et l'Affaire Dreyfus, 1898-1908. Paris : Champ Vallon, 2009.

VASSELIN, Martine. «Le corps dénudé de la Vérité ». Rives nord-méditerranéennes, 30 (2008). http://rives. revues.org/2363

VOUILLOUX, Bernard. «Introduction. Le retour de l'allégorie » et « Entre textes et images. Le tournant moderne de l'allégorie ». In Déclins de l'allégorie ?. Ed. Bernard Vouilloux, Modernités, n²2. Bordeaux : PU de Bordeaux, 2006. 7-16 et 17-83.

— «escriptions ». Le Tournant « artiste » de la littérature française. Paris : Hermann, 2011. 31-75.

— « Lire, voir. La co-implication du verbal et du visuel ». Textimage. Revue d'étude du dialogue texte-image. Dir. Barre, Aurélie et Leplatre, Olivier, « Varia n³ » (2013). http://www.revue-textimage.com/07_varia_3/ vouilloux $1 . h$ tml

WINOCK, Michel. Le siècle des intellectuels. Paris : Le Seuil, 1999.

ZOLA, Émile. La Vérité en marche. Textes sur l'affaire Dreyfus. 1901. Ed. Vincent Duclert. Paris : Texto, 2013. 